\title{
New Bounds for PMAC, TMAC, and XCBC
}

\author{
Kazuhiko Minematsu ${ }^{1,2}$ and Toshiyasu Matsushima ${ }^{2}$ \\ ${ }^{1}$ NEC Corporation, 1753 Shimonumabe, Nakahara-Ku, Kawasaki, Japan \\ k-minematsu@ah.jp.nec.com \\ ${ }^{2}$ Waseda University, 3-4-1 Okubo Shinjuku-ku Tokyo, Japan
}

\begin{abstract}
We provide new security proofs for PMAC, TMAC, and $\mathrm{XCBC}$ message authentication modes. The previous security bounds for these modes were $\sigma^{2} / 2^{n}$, where $n$ is the block size in bits and $\sigma$ is the total number of queried message blocks. Our new bounds are $\ell q^{2} / 2^{n}$ for PMAC and $\ell q^{2} / 2^{n}+\ell^{4} q^{2} / 2^{2 n}$ for TMAC and XCBC, where $q$ is the number of queries and $\ell$ is the maximum message length in $n$-bit blocks. This improves the previous results under most practical cases, e.g., when no message is exceptionally long compared to other messages.
\end{abstract}

\section{Introduction}

Message authentication code (MAC) is a symmetric-keyed function used for ensuring the authenticity of messages. Many studies have been done on MACs built using blockciphers (i.e., MAC modes of operation) including the CBC-MAC and its variants. The theoretical security of stateless (i.e., no counter or nonce is used) MAC mode $F\left[E_{K}\right]$ using blockcipher $E_{K}$ can be measured using the maximum advantage of an adversary trying to distinguish $F\left[E_{K}\right]$ from the random oracle, which provides an independent and uniform output for any distinct input, using a chosen-plaintext attack (CPA). Typically, the key task in proving the maximum advantage is to prove the maximum information-theoretic (IT) advantage for the target MAC, where the adversary has infinite computational power and the MAC is built using the uniform random permutation (URP), which is the ideal functionality of a blockcipher. Improving the maximum IT-advantage is important, because it will contribute to better understanding of the target function and to expanding the scope of application.

Bellare, Pietrzak, and Rogaway [5] analyzed the IT-advantage for the CBCMAC and the encrypted CBC-MAC called EMAC 2. Neglecting constants, the previous EMAC bound using the $n$-bit $\operatorname{URP}$ was $\ell^{2} q^{2} / 2^{n}$ [7 for any $(q, \ell)$ CPA, which uses $q$ chosen messages with lengths less than $n \ell$ bits. Bellare et al. investigated whether this could be improved, particularly with respect to $\ell$. They proved the improved bound $\mathrm{d}(\ell) q^{2} / 2^{n}+\ell^{4} q^{2} / 2^{2 n}$, where $\mathrm{d}(\ell)$ is a function that grows very slowly with $\ell$ (see Sect.4). A similar result was obtained for CBC-MAC for prefix-free messages. Recently, Pietrzak 18, proved EMAC bound $q^{2} / 2^{n}$ for a range of $\left(q, \ell\right.$ ) (in fact, the result was $q^{2} / 2^{n}+\ell^{8} q^{2} / 2^{2 n}$ for any $q \geq \ell^{2}$ ).

Given these findings, it is quite natural to ask if similar improvements can be obtained for modes other than EMAC, especially more sophisticated ones.

A. Biryukov (Ed.): FSE 2007, LNCS 4593, pp. 434451 2007.

(C) International Association for Cryptologic Research 2007 
EMAC uses two blockcipher keys, and only messages with a length multiple of $n$ are supported. In this paper, we describe several MAC modes and provide new security bounds for them. Our first target is PMAC, which was proposed by Black and Rogaway [7] and Rogaway [19. It is a one-key MAC; i.e., the MAC function uses one blockcipher key, and messages of any lengths are supported, and is fully parallelizable. The original security bound was $\sigma^{2} / 2^{n}$ [719], where $\sigma$ is the total number of queried message blocks, which immediately implies $\ell^{2} q^{2} / 2^{n}$ for any $(q, \ell)-\mathrm{CPA}$, as $\sigma \leq \ell q$ holds. Here, we demonstrate a new bound $\ell q^{2} / 2^{n}$ by taking an approach different from that of the previous proof.

We also provide new bounds for two successors of EMAC called TMAC 13 and XCBC [7. Like EMAC, they are based on CBC-MAC. However, they do not use two blockcipher keys, and can efficiently handle messages of arbitrary length. Our bounds are obtained by combining our PMAC proof technique and the CBC-MAC collision analysis provided by Bellare et al.[5]. For TMAC and XCBC, the previous bounds are $\sigma^{2} / 2^{n}$ shown by Iwata and Kurosawa 10, and our bound is $\ell q^{2} / 2^{n}+\ell^{4} q^{2} / 2^{2 n}$. We also investigated OMAC [11] (i.e., CMAC [1]), which is an optimized version of TMAC and XCBC. Although some part of TMAC proof can also be applied to OMAC, we could not obtain a new bound at this moment. The analysis of OMAC is briefly described in Sect.5.

We have to emphasize that our results are not always better than the previous ones. Since all of our targets have $\sigma^{2} / 2^{n}$ bounds, ours are worse if message length distribution is heavily biased to the left, e.g., one $\ell$-block message and $(q-1)$ one-block messages. For other cases, ours are better. A detailed comparison is given in Sect.5.

\section{Preliminaries}

Notation. $\{0,1\}$ and $\{0,1\}^{n}$ are denoted by $\Sigma$ and $\Sigma^{n}$. The set of $i$-bit sequences for all $i=1, \ldots, n$ is denoted by $\Sigma \leq n \stackrel{\text { def }}{=} \bigcup_{i=1, \ldots n} \Sigma^{i} .\left(\Sigma^{n}\right) \leq m$ is the set of binary sequences with lengths that are a multiple of $n$ and at most $n m$. $\Sigma^{*}$ is the set of all finite-length bit sequences. The bit length of $x$ is denoted by $|x|$.

The $n$-bit uniform random permutation (URP), denoted by $\mathrm{P}_{n}$, is a random permutation with a uniform distribution over all permutations on $\Sigma^{n}$. The random oracle $(\mathrm{RO})$, which has $n$-bit output and is denoted by $\mathrm{O}_{n}$, is a random function that accepts any $x \in \Sigma^{*}$ and outputs independent and uniformly random $n$-bit values for any distinct inputs. For any two colliding inputs, RO outputs the same value.

Field With $2^{n}$ POINTS. We consider the elements of field $\operatorname{GF}\left(2^{n}\right)$ as $n$-bit coefficient vectors of the polynomials in the field. We represent $n$-bit coefficient vectors by integers $0,1, \ldots, 2^{n}-1$, e.g., 2 corresponds to coefficient vector (00 . . 010), which corresponds to $\mathrm{x}$ in the polynomial representation, and 3 denotes $(00 \ldots 011)$, which corresponds to $\mathrm{x}+1$. For any $x, y \in \Sigma^{n}, x y$ denotes the field multiplication of two elements represented by $x$ and $y$. For simplicity, we assume $n=128$ throughout the paper. 
SECURITY NOtions. We used the standard security notion for symmetric cryptography 349].

Definition 1. Let $F$ and $G$ be two random (here, random means it is probabilistic) functions. The oracle has implemented $H$, which is identical to one of $F$ or $G$. An adversary, $A$, guesses if $H$ is $F$ or $G$ using a $\theta$-chosen-plaintext attack $(\theta-C P A)$, where $\theta$ is a list of parameters, such as the number of queries. The maximum advantage in distinguishing $F$ from $G$ is defined as

$$
\operatorname{Adv}_{F, G}^{\text {cpa }}(\theta) \stackrel{\text { def }}{=} \max _{A: \theta-\mathrm{CPA}}\left|\operatorname{Pr}\left[A^{F}=1\right]-\operatorname{Pr}\left[A^{G}=1\right]\right|,
$$

where $A^{F}=1$ denotes that $A$ 's guess is 1 , which indicates one of $F$ or $G$. The probabilities are determined by the randomness of $F$ or $G$ and $A$.

The Goal of Our Analysis. In this paper, we consider only the informationtheoretic security, where the adversary has infinite computational power (thus $\theta$ contains no computational restrictions), and the target is realized by the ideal $n$-bit blockcipher, i.e., $\mathrm{P}_{n}$. In many cases, including ours, once the informationtheoretic security is proved, the computational counterpart, where the adversary is computationally restricted and a real blockcipher is used, is quite easy.

Our target modes are stateless and variable-input-length (VIL) functions with $n$-bit output (VIL means that the domain is $\Sigma^{*}$ ). Therefore, for mode $F\left[E_{K}\right]$, where $E_{K}$ is a blockcipher, we evaluate $\operatorname{Adv}_{F\left[\mathrm{P}_{n}\right]}^{\text {vilqrf }}(\theta) \stackrel{\text { def }}{=} \operatorname{Adv}_{F\left[\mathrm{P}_{n}\right], \mathrm{O}_{n}}^{\text {cpa }}(\theta)$. vilqre denotes a VIL quasi-random function [15] that cannot be informationtheoretically distinguished from RO without a negligibly small success probability. If $\operatorname{Adv}_{F\left[\mathrm{P}_{n}\right]}^{\text {vilqrf }}(\theta)$ is small, the maximum success probability of a MAC forgery for all $\theta$-CPAs against $F\left[\mathrm{P}_{n}\right]$ is also small (e.g., see Proposition 2.7 of [3]). In this paper, $\theta$ contains one of two additional parameters in addition to the number of queries, $q$ : the total number of $n$-bit blocks for all $q$ queries, $\sigma$, and the maximum length of a query (in $n$-bit blocks), $\ell$. We focus on the $\theta=(q, \ell)$ case.

\section{PMAC}

\subsection{Description and Previous Security Proof}

PMAC has two versions; we focus on the later version [12 19. We call it simply "PMAC". The main idea of PMAC is as follows.

Lemma 1. (Proposition 5 of [19]) Assume that the representation of $\mathrm{GF}\left(2^{n}\right)$ $(n=128)$ is based on the lexicographically first primitive polynomial (see [19] for details). Let $\mathbb{I}=\left\{1, \ldots, 2^{n / 2}\right\}$ and $\mathbb{J}=\{0,1,2\}$ be the set of integers used as indices for distinct elements ("bases") of $\mathrm{GF}\left(2^{n}\right)$. Then, for any $(\alpha, \beta),\left(\alpha^{\prime}, \beta^{\prime}\right) \in \mathbb{I} \times \mathbb{J}$, $2^{\alpha} 3^{\beta} \neq 2^{\alpha^{\prime}} 3^{\beta^{\prime}}$ holds if $(\alpha, \beta) \neq\left(\alpha^{\prime}, \beta^{\prime}\right)$ hold 1 .

\footnotetext{
${ }^{1}$ Actually, Proposition 5 of 19 proved this for a wider range of indices. The original PMAC uses $\mathbb{J}=\{2,3,4\}$ instead of $\{0,1,2\}$, so our PMAC definition is slightly different from the original. However, the security proofs are essentially the same.
} 

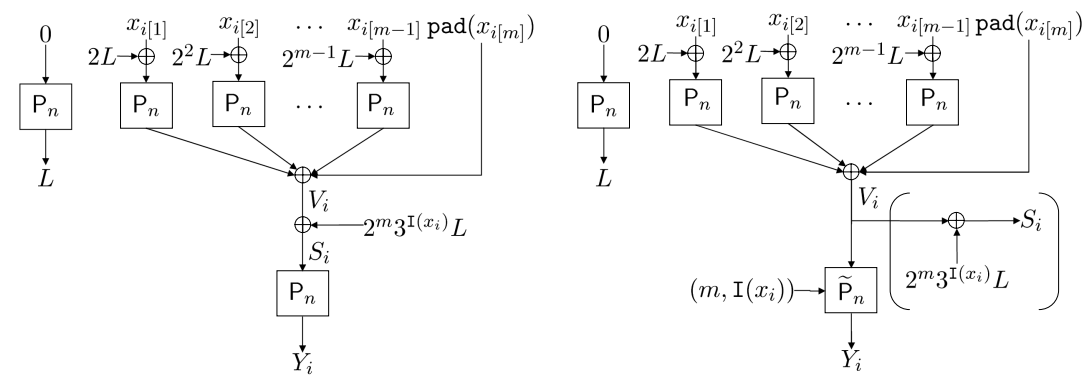

Fig. 1. $\mathrm{PMAC}\left[\mathrm{P}_{n}\right]$ (left) and the modified PMAC (MPMAC) (right)

Multiplication of a constant and a variable is generally much simpler than multiplication of variables. For example, multiplication with 2 (i.e., a doubling operation) requires a bit shift followed by a conditional xor. Therefore, computation of $2^{\alpha} 3^{\beta}$ from $2^{\alpha-1} 3^{\beta}$ or $2^{\alpha} 3^{\beta-1}$ is significantly faster than one blockcipher invocation. The idea of PMAC [19], called the "powering-up construction", is to use $2^{\alpha} 3^{\beta}$ as a masking value for every blockcipher input, incrementing $\alpha$ or $\beta$.

For blockcipher $E_{K}$, PMAC is defined as follows. We say "partition $x \in \Sigma^{*}$ into $\left(x_{[1]}, \ldots, x_{[m]}\right) "$ to set $m=\|x\|_{n} \stackrel{\text { def }}{=} \max \{\lceil|x| / n\rceil, 1\}$ and $x=\left(x_{[1]}, \ldots, x_{[m]}\right)$ with $x_{[i]} \in \Sigma^{n}$ for $i=1, \ldots, m-1$ and $x_{[m]} \in \Sigma^{\leq n}$. First, compute $L=E_{K}(0)$, where 0 corresponds to the all-zero $n$-bit sequence, in the preprocessing. Then, for input $x \in \Sigma^{*}$, partition it into $\left(x_{[1]}, \ldots, x_{[m]}\right)$. The tag for $x$ is $Y=E_{K}$ (Psum $\oplus$ $\left.\operatorname{pad}\left(x_{[m]}\right) \oplus 2^{m} 3^{\mathrm{I}(x)}\right)$, where Psum $=\bigoplus_{\alpha=1}^{m-1} E_{K}\left(x_{[\alpha]} \oplus 2^{\alpha} L\right)$ if $m>1$, and if $m=1$, Psum $=0$. Here, $\mathrm{I}(x)=1$ if $|x|$ is a multiple of $n$ and $\mathrm{I}(x)=2$ otherwise, and $\operatorname{pad}\left(x_{[m]}\right)=x_{[m]}$ if $\left|x_{[m]}\right|=n$ and $\operatorname{pad}\left(x_{[m]}\right)=x_{[m]} \| 10^{*}$ otherwise, where $x_{[m]} \| 10^{*}$ is a concatenation of $x_{[m]}$ and the $\left(n-\left|x_{[m]}\right|\right)$-bit sequence $(100 \ldots 0)$.

Rogaway [19] proved the security of PMAC, which is as follows.

Theorem 1. (Corollary 17 of [19]) Let $\mathrm{PMAC}\left[\mathrm{P}_{n}\right]$ be the PMAC using $\mathrm{P}_{n}$ (see the left of Fig. 11). We then have $\operatorname{Adv}_{\left.\mathrm{PMAC}^{\mathrm{vilqu}} \mathrm{P}_{n}\right]}^{\text {vila }}(q, \sigma) \leq 5.5 \sigma^{2} / 2^{n}$ and $\operatorname{Adv}_{\operatorname{PMAC}\left[\mathrm{P}_{n}\right]}^{\mathrm{vilquf}}(q, \ell) \leq 5.5 \ell^{2} q^{2} / 2^{n}$, where $q, \sigma$, and $\ell$ are as defined in Sect. Q

Corollary 17 of 19 only proved the first claim. The second follows from the first and $\sigma \leq \ell q$.

\subsection{New Security Bound for PMAC}

Our security bound of PMAC is the following. The proof will be provided later.

Theorem 2. Let $\mathrm{PMAC}\left[\mathrm{P}_{n}\right]$ be the PMAC using $\mathrm{P}_{n}$. We then have

$$
\operatorname{Adv}_{\operatorname{PMAC}\left[\mathrm{P}_{n}\right]}^{\mathrm{vilqrf}}(q, \ell) \leq \frac{5 \ell q^{2}}{2^{n}-2 \ell}
$$

From this theorem, we have $\operatorname{Adv}_{\operatorname{PMAC}_{\left[\mathrm{P}_{n}\right]}^{\text {vilqrf }}}(q, \ell) \leq 10 \ell q^{2} / 2^{n}$ if $\ell \leq 2^{n-2}$. 
Notation for Proof. Since we use Maurer's methodology 2 (e.g., see [15]) to make our proofs intuitive and simple, we briefly describe his notation. For completeness, part of his results that we used for our proof is cited in Appendix A Consider event $a_{i}$ defined for $i$ input/output pairs, and possibly some internal variables, of random function $F$. Let $\overline{a_{i}}$ be the negation of $a_{i}$. We assume $a_{i}$ is monotone; i.e., $a_{i}$ never occurs if $\overline{a_{i-1}}$ occurs. For instance, $a_{i}$ is monotone if it indicates that all $i$ outputs are distinct. An infinite sequence of monotone events, $\mathcal{A}=a_{0} a_{1} \ldots$, is called a monotone event sequence (MES) [15]. Here, $a_{0}$ denotes some tautological event. Note that $\mathcal{A} \wedge \mathcal{B}=\left(a_{0} \wedge b_{0}\right)\left(a_{1} \wedge b_{1}\right) \ldots$ is an MES if $\mathcal{A}=a_{0} a_{1} \ldots$ and $\mathcal{B}=b_{0} b_{1} \ldots$ are both MESs. For any sequence of variables, $X_{1}, X_{2}, \ldots$, let $X^{i}$ denote $\left(X_{1}, \ldots, X_{i}\right)$. We use $\operatorname{dist}\left(X^{i}\right)$ (or, equivalently, $\operatorname{dist}\left(\mathbf{X}^{(i)}\right)$, where $\mathbf{X}^{(i)}$ is set $\left.\left\{X_{j}\right\}_{j=1, \ldots, i}\right)$ to denote an event where $X_{1}, X_{2}, \ldots, X_{i}$ are distinct.

Let MESs $\mathcal{A}$ and $\mathcal{B}$ be defined for two random functions, $F$ and $G$, respectively. Let $X_{i}$ and $Y_{i}$ be the $i$-th input and output. Let $P^{F}$ be the probability space defined by $F$. For example, $P_{Y_{i} \mid X^{i} Y^{i-1}}^{F}\left(y^{i}, x^{i}\right)$ means $\operatorname{Pr}\left[Y_{i}=y_{i} \mid X^{i}=x^{i}, Y^{i-1}=\right.$ $\left.y^{i-1}\right]$, where $Y_{j}=F\left(X_{j}\right)$ for $j \geq 1$.

Definition 2. Let $\theta$ contain $q$. For $M E S \mathcal{A}$ defined for $F, \nu_{\theta}\left(F, \overline{a_{q}}\right)$ denotes the maximum probability of $\overline{a_{q}}$ for any $\theta-C P A$ that interacts with $F$. Similarly, $\mu_{\theta}\left(F, \overline{a_{q}}\right)$ denotes the maximum probability of $\overline{a_{q}}$ for any non-adaptive $\theta-C P A$. For $\theta=(q, \ell)$, they are abbreviated to $\nu_{\ell}\left(F, \overline{a_{q}}\right)$ and $\mu_{\ell}\left(F, \overline{a_{q}}\right)$. If $\theta=q$, the subscript is omitted, e.g., we write $\nu\left(F, \overline{a_{q}}\right)$.

Here, $\mu_{\theta}\left(F, \overline{a_{q}}\right)$ can be rewritten as $\max _{x^{q}} P_{\overline{a_{q}} \mid X^{q}}^{F}\left(x^{q}\right)$, where the maximum is taken for all (non-adaptively chosen) $x^{q}$ satisfying $\theta$ (e.g., if $\theta=(q, \ell),\left|x_{i}\right| \leq n \ell$ for all $i \leq q)$, hereafter abbreviated to $\max _{X^{q}} P \frac{F}{a_{q}} \mid X^{q}$.

Analysis of PHASH. Proving Theorem 2 requires an analysis of the messagehashing part of PMAC $\left[\mathrm{P}_{n}\right]$, which we call PHASH. For $x=\left(x_{[1]}, \ldots, x_{[m]}\right) \in$ $\left(\Sigma^{n}\right)^{m}$, it is defined as:

$$
\operatorname{PHASH}(x) \stackrel{\text { def }}{=} \bigoplus_{i=1, \ldots, m} \mathrm{P}_{n}\left(x_{[i]} \oplus 2^{i} L\right) \text {, where } L=\mathrm{P}_{n}(0) .
$$

Lemma 2. For any $x=\left(x_{[1]}, \ldots, x_{[m]}\right) \in\left(\Sigma^{n}\right)^{m}$ and $x^{\prime}=\left(x_{[1]}^{\prime}, \ldots, x_{\left[m^{\prime}\right]}^{\prime}\right) \in$ $\left(\Sigma^{n}\right)^{m^{\prime}}, x \neq x^{\prime}$, and for any $f: \Sigma^{n} \rightarrow \Sigma^{n}$, we have

$$
\begin{array}{r}
\operatorname{Pr}\left[\operatorname{PHASH}(x) \oplus \operatorname{PHASH}\left(x^{\prime}\right)=f(L)\right] \leq \frac{m+m^{\prime}}{2^{n}}+\frac{1}{2^{n}-\left(m+m^{\prime}\right)} \text {, and } \\
\operatorname{Pr}[\operatorname{PHASH}(x)=f(L)] \leq \frac{m}{2^{n}}+\frac{1}{2^{n}-m} \text {, where } L=\mathrm{P}_{n}(0) .
\end{array}
$$

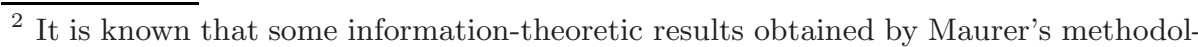
ogy can not be converted into computational ones (for instance, see [16[17]). However, we do not encounter such difficulties in this paper.
} 
Proof. We only prove Eq. (11) as Eq. (2) can be similarly proved. Fix $x$ and $x^{\prime}$. Let $U_{i}=x_{[i]} \oplus 2^{i} L$ for $i=1, \ldots, m$, and $U_{i}=x_{[i-m]}^{\prime} \oplus 2^{i-m} L$ for $i=m+1, \ldots, m+m^{\prime}$. Then, $\operatorname{PHASH}(x) \oplus \operatorname{PHASH}\left(x^{\prime}\right)$ equals Sum $\stackrel{\text { def }}{=} \mathrm{P}_{n}\left(U_{1}\right) \oplus \ldots \oplus \mathrm{P}_{n}\left(U_{m+m^{\prime}}\right)$. Let $\mathbf{U}=\left\{U_{1}, \ldots, U_{m+m^{\prime}}\right\} \backslash \mathbf{U}_{\text {coll }}$, where $\mathbf{U}_{\text {coll }}$ is the set of all trivial collisions, e.g., $U_{1}$ and $U_{1+m}$ when $x_{[1]}=x_{[1]}^{\prime}$. Note that $\mathbf{U}$ can not be the empty set as $x \neq$ $x^{\prime}$. For simplicity, we assume no trivial collision (thus $\mathbf{U}=\left\{U_{1}, \ldots, U_{m+m^{\prime}}\right\}$ ), however the following analysis works even if some trivial collisions exist. For index subset $\left\{i_{1}, \ldots, i_{k}\right\} \subseteq\{1, \ldots, q\}, \mathbf{U}_{\text {sub }}=\left\{U_{i_{j}}\right\}_{j=1, \ldots, k}$ is an equivalent set if $U_{i_{1}}=U_{i_{2}}=\cdots=U_{i_{k}}$ and $U_{i_{1}} \neq U_{h}$ for all $h \notin\left\{i_{1}, \ldots, i_{k}\right\}$. Here, the sum of all equivalent sets is a decomposition of $\mathbf{U}$. Whether $\mathbf{U}_{\text {sub }}$ is an equivalent set or not depends on the value of $L$. If $k$ is odd (even), we say $\mathbf{U}_{\text {sub }}$ is an odd (even) equivalent set. Let odd $k$ be the event such that there are $k$ odd equivalent sets having non-zero values (the value of an equivalent set is the value of its members). We have

$$
\begin{aligned}
& \operatorname{Pr}\left[\operatorname{Sum}=f(L) \mid \operatorname{odd}_{k}\right] \\
& \leq \max _{c \text { satisfies odd }} \operatorname{Pr}\left[\mathrm{P}_{n}\left(u_{1}(c)\right) \oplus \ldots \oplus \mathrm{P}_{n}\left(u_{m+m^{\prime}}(c)\right)=f(c) \mid \operatorname{odd}_{k}, L=c\right],
\end{aligned}
$$

where $u_{i}(c)$ is $x_{[i]} \oplus 2^{i} c$ for $i=1, \ldots, m$ and $x_{[i-m]}^{\prime} \oplus 2^{i-m} c$ for $i=m+$ $1, \ldots, m+m^{\prime}$. In Eq. (3), note that $\mathrm{P}_{n}\left(u_{i}(c)\right)$ is canceled out if $u_{i}(c)$ is in an even equivalent set. Therefore, given $L=c$ and odd $k$ for some $k>0, \mathrm{P}_{n}\left(u_{1}(c)\right) \oplus \ldots \oplus$ $\mathrm{P}_{n}\left(u_{m+m^{\prime}}(c)\right)$ is either the sum of $k$ URP outputs for $k$ non-zero distinct inputs or the sum of $c$ and $k$ URP outputs for $k$ non-zero distinct inputs (note that $\operatorname{odd}_{k}$ does not exclude an odd equivalent set with value 0$)$. Then, the property of $\mathrm{P}_{n}$ shows that, for any non-zero distinct $k$ inputs, $z_{1}, \ldots, z_{k}$,

$$
\begin{aligned}
& \operatorname{Pr}\left[\mathrm{P}_{n}\left(z_{1}\right) \oplus \mathrm{P}_{n}\left(z_{2}\right) \oplus \ldots \oplus \mathrm{P}_{n}\left(z_{k}\right)=f(c) \mid \mathrm{P}_{n}(0)=c\right] \\
& =\sum_{c_{1}, \ldots, c_{k}, \operatorname{dist}\left(\left\{c_{1}, \ldots, c_{k}, c\right\}\right), c_{1} \oplus \ldots \oplus c_{k}=f(c)} \operatorname{Pr}\left[\mathrm{P}_{n}\left(z_{1}\right)=c_{1}, \ldots, \mathrm{P}_{n}\left(z_{k}\right)=c_{k} \mid \mathrm{P}_{n}(0)=c\right] \\
& =\frac{\left|\left\{\left(c_{1}, \ldots, c_{k}\right): \operatorname{dist}\left(\left\{c_{1}, \ldots, c_{k}, c\right\}\right), c_{1} \oplus \ldots \oplus c_{k}=f(c)\right\}\right|}{\left(2^{n}-1\right) \cdots \cdots\left(2^{n}-k\right)} \leq \frac{1}{2^{n}-k}
\end{aligned}
$$

holds, where the inequality holds since $c_{k}$ is uniquely determined (or does not exist) if $c_{1}, \ldots, c_{k-1}$ are fixed. From Eqs. (3) and (4), we obtain

$$
\operatorname{Pr}\left[\text { Sum }=f(L) \mid \text { odd }_{k}\right] \leq \frac{1}{2^{n}-k} \text { for any } 0<k \leq m+m^{\prime} \text { and for any } f .
$$

Next, we analyze $\operatorname{Pr}\left[\operatorname{odd}_{0}\right]$. We have

$$
\begin{aligned}
\operatorname{Pr}\left[\operatorname{odd}_{0}\right] & =\operatorname{Pr}\left[\operatorname{odd}_{0}, U_{1} \notin\left\{U_{2}, \ldots, U_{m+m^{\prime}}\right\}\right] \\
& +\operatorname{Pr}\left[\operatorname{odd}_{0}, U_{1}=U_{j} \text { for some } j=2, \ldots, m+m^{\prime}\right] \\
& \leq \operatorname{Pr}\left[U_{1}=0\right]+\sum_{j=2, \ldots, m+m^{\prime}} \operatorname{Pr}\left[U_{1}=U_{j}\right] \leq\left(m+m^{\prime}\right) \frac{1}{2^{n}},
\end{aligned}
$$


where the first inequality holds since if $U_{1}$ is unique (i.e., $U_{1}$ is in an odd equivalent set) and odd 0 holds, $U_{1}$ must be 0 . The second holds since both $U_{1}$ and $U_{1} \oplus U_{j}$ for any $j \neq 1$ are permutations of $L$ from Lemma 1) From Eqs. (5) and (6), we obtain

$$
\begin{aligned}
\operatorname{Pr}[\text { Sum }=f(L)] & =\sum_{k=0, \ldots, m+m^{\prime}} \operatorname{Pr}\left[\text { Sum }=f(L) \mid \text { odd }_{k}\right] \cdot \operatorname{Pr}\left[\text { odd }_{k}\right] \\
& \leq \operatorname{Pr}\left[\text { odd }_{0}\right]+\sum_{k=1}^{m+m^{\prime}-1} \frac{\operatorname{Pr}\left[\text { odd }_{k}\right]}{2^{n}-k}+\frac{1-\sum_{k=0}^{m+m^{\prime}-1} \operatorname{Pr}\left[\text { odd }_{k}\right]}{2^{n}-\left(m+m^{\prime}\right)} \\
& \leq \operatorname{Pr}\left[\text { odd }_{0}\right]+\frac{1}{2^{n}-\left(m+m^{\prime}\right)} \leq \frac{m+m^{\prime}}{2^{n}}+\frac{1}{2^{n}-\left(m+m^{\prime}\right)} .
\end{aligned}
$$

This concludes the proof of Lemma 2 .

Proof of Theorem [2, First, we introduce the tweakable[14] $n$-bit URP, $\widetilde{\mathrm{P}}_{n}$. It has tweak space $\mathcal{T}=\mathbb{I} \times \mathbb{J}^{\prime}$, where $\mathbb{I}=\left\{1, \ldots, 2^{n / 2}\right\}$ and $\mathbb{J}^{\prime}=\{1,2\}$. It consists of $|\mathcal{T}|$ independent $n$-bit URPs; $\widetilde{\mathrm{P}}_{n}(t, x)$ is the output of an $n$-bit URP indexed by $t \in \mathcal{T}$ and having input $x \in \Sigma^{n}$. Using $\widetilde{\mathrm{P}}_{n}$ and $\mathrm{P}_{n}$, independent of $\widetilde{\mathrm{P}}_{n}$, we define the modified PMAC (MPMAC) as follows. First, compute $L=\mathrm{P}_{n}(0)$. For input $x \in \Sigma^{*}=\left(x_{[1]}, \ldots, x_{[m]}\right)$, compute Psum using PHASH (i.e., Psum = $\operatorname{PHASH}(\widehat{x})$, where $\widehat{x}=\left(x_{[1]}, \ldots, x_{[m-1]}\right)$, if $m>1$, and Psum $=0$ otherwise). The tag is $Y=\widetilde{\mathrm{P}}_{n}\left((m, \mathrm{I}(x))\right.$, Psum $\left.\oplus \operatorname{pad}\left(x_{[m]}\right)\right)$. Here, $(m, \mathrm{I}(x))$ is the tweak. Note that a tweak is a function of $x$.

Proof IDEA. Since the advantage is the absolute difference between two prob-

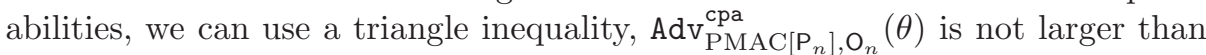
$\operatorname{Adv}_{\mathrm{PMAC}\left[\mathrm{P}_{n}\right], H}^{\mathrm{cpa}}(\theta)+\operatorname{Adv}_{H, \mathrm{O}_{n}}^{\mathrm{cpa}}(\theta)$ for any VIL function $H$, and for any $\theta$. Here, $H$ is an intermediate function. Theorem 1 was derived using "PMAC with an ideal tweakable blockcipher", which invokes an independent URP for each message block in the message-hashing part as well as in the finalization, as the intermediate function. Here, our proof uses MPMAC as the intermediate function.

We start by proving the advantage between PMAC $\left[\mathrm{P}_{n}\right]$ and MPMAC, which requires defining some random variables. Let $X_{i} \in \Sigma^{*}$ be the $i$-th query of the adversary. If $m=\left\|X_{i}\right\|_{n}$, we write $X_{i}=\left(X_{i[1]}, \ldots, X_{i[m]}\right)$. Note that $X_{i}$ is a random variable, and its distribution is determined by the adversary and the target MAC. Fixed queries (and other random variables) are written in lower case, e.g., $x_{i}=\left(x_{i[1]}, \ldots, x_{i[m]}\right)$. For PMAC $\left[\mathrm{P}_{n}\right]$, let $\mathbf{M}^{(q)}\left(\mathbf{C}^{(q)}\right)$ be the set of inputs (outputs) to $\mathrm{P}_{n}$ generated in the PHASH for all $q$ queries. We do not include the result of preprocessing, i.e., $L=\mathbf{P}_{n}(0)$, in $\mathbf{M}^{(q)}$ and $\mathbf{C}^{(q)}$. We also define $Y_{i} \in \Sigma^{n}$ as the $i$-th tag, and $\mathbf{Y}^{(q)} \stackrel{\text { def }}{=}\left\{Y_{i}\right\}_{i=1, \ldots, q}$. If $m=\left\|X_{i}\right\|_{n}>1$, we define $V_{i}$ as the XOR of the $i$-th PHASH output and $\operatorname{pad}\left(X_{i[m]}\right)$. If $m=1, V_{i}=\operatorname{pad}\left(X_{i}\right)$. Moreover, $S_{i} \stackrel{\text { def }}{=} V_{i} \oplus 2^{m} 3^{\mathrm{I}\left(X_{i[m]}\right)} L$, and $\mathbf{S}^{(q)} \stackrel{\text { def }}{=}\left\{S_{i}\right\}_{i=1, \ldots, q}$. Thus, in PMAC $\left[\mathrm{P}_{n}\right]$, $Y_{i}=\mathrm{P}_{n}\left(S_{i}\right)$. These variables are similarly defined for MPMAC except $Y_{i}$; in MPMAC, $Y_{i}$ is $\widetilde{\mathrm{P}}_{n}\left(\left(m, \mathrm{I}\left(X_{i[m]}\right)\right), V_{i}\right)$ when the $i$-th query has $m$ blocks. Also, $S_{i}$ is defined as a dummy variable in MPMAC. See Fig.1 for reference. 
Lemma 3. Let event $a_{q} \stackrel{\text { def }}{=}\left[\mathbf{M}^{(q)} \cap \mathbf{S}^{(q)}=\emptyset\right] \wedge\left[\operatorname{dist}\left(\mathbf{S}^{(q)}\right)\right] \wedge\left[0 \notin \mathbf{M}^{(q)} \cup \mathbf{S}^{(q)}\right]$. Moreover, $b_{q} \stackrel{\text { def }}{=}\left[\operatorname{dist}\left(\mathbf{Y}^{(q)}\right)\right], d_{q} \stackrel{\text { def }}{=}\left[\mathbf{C}^{(q)} \cap \mathbf{Y}^{(q)}=\emptyset\right]$, and $e_{q} \stackrel{\text { def }}{=}\left[L \notin \mathbf{Y}^{(q)}\right]$, where $L=\mathrm{P}_{n}(0)$. We then have

$$
\begin{aligned}
\operatorname{Adv}_{\mathrm{PMAC}\left[\mathrm{P}_{n}\right], \operatorname{MPMAC}}^{\mathrm{cpa}}(q, \ell) & \leq \nu_{\ell}\left(\mathrm{MPMAC}, \overline{a_{q} \wedge b_{q} \wedge d_{q} \wedge e_{q}}\right) \\
& \leq \nu_{\ell}\left(\mathrm{MPMAC}, \overline{a_{q} \wedge b_{q}}\right)+\nu_{\ell}\left(\mathrm{MPMAC}, \overline{d_{q} \wedge e_{q}}\right) \\
& \leq \frac{1}{2^{n}-2 \ell}\left((4 \ell-2.5) q^{2}+1.5 q\right) .
\end{aligned}
$$

Proof. (of Lemma 3i) The first and second inequalities are derived from Maurer's methodology. See Appendix $\mathrm{B}$ for the proof. In the following, we prove the third. ANALYSIS FOR $\nu_{\ell}\left(\mathrm{MPMAC}, \overline{a_{q} \wedge b_{q}}\right)$. We use the following lemma. The proof is in Appendix C.

\section{Lemma 4}

$\nu_{\ell}\left(\mathrm{MPMAC}, \overline{a_{q} \wedge b_{q}}\right)=\mu_{\ell}\left(\mathrm{MPMAC}, \overline{a_{q} \wedge b_{q}}\right)=\max _{X^{q}} P_{\overline{a_{q}} \mid X^{q}}^{\mathrm{MPMAC}}+\max _{X^{q}} P_{\overline{b_{q}} \mid a_{q} X^{q}}^{\mathrm{MPMAC}}$,

where the maximums are taken for all $X^{q}=x^{q}$ with $\left|x_{i}\right| \leq n \ell$ for all $i$.

Note that $\max _{X^{q}} P_{\overline{a_{q}} \mid X^{q}}^{\mathrm{MPMAC}}$ denotes $\max _{x^{q}} P_{\overline{a_{q}} \mid X^{q}}^{\mathrm{MPMAC}}\left(x^{q}\right)$. Let $\mathbf{M}_{i}$ denote the input set to $\mathrm{P}_{n}$ that occur in the $i$-th PHASH call, except the all-zero input used to obtain $L$. Note that $\mathbf{M}^{(q)}=\mathbf{M}_{1} \cup \cdots \cup \mathbf{M}_{q}$. For $i=1, \ldots, q$, we have

$\chi_{1} \stackrel{\text { def }}{=} \operatorname{dist}\left(\mathbf{S}^{(q)}\right), \quad \chi_{2, i} \stackrel{\text { def }}{=}\left[S_{i} \notin \mathbf{M}^{(q)}\right], \quad \chi_{3, i} \stackrel{\text { def }}{=}\left[S_{i} \neq 0\right]$, and $\quad \chi_{4, i} \stackrel{\text { def }}{=}\left[0 \notin \mathbf{M}_{i}\right]$.

Note that $\overline{a_{q}} \equiv \overline{\chi_{1} \wedge \chi_{2} \wedge \chi_{3} \wedge \chi_{4}}$ where $\chi_{i} \stackrel{\text { def }}{=} \chi_{i, 1} \wedge \cdots \wedge \chi_{i, q}$ for $i=2,3,4$. Using the union bound and its variant, we have

$$
\begin{aligned}
\max _{X^{q}} P_{\overline{a_{q}} \mid X^{q}}^{\mathrm{MPMAC}} & \leq \max _{X^{q}} P_{\bar{\chi}_{1} \mid X^{q}}^{\mathrm{MPMAC}} \\
& +\sum_{i=1, \ldots, q}\left(\max _{X^{q}} P \frac{\mathrm{MPMAC}}{\chi_{2, i} \mid \chi_{4, i}, X^{q}}+\max _{X^{q}} P \frac{\mathrm{MPMAC}}{\bar{\chi}_{3, i} \mid \chi_{4, i}, X^{q}}+\max _{X^{q}} P \frac{\mathrm{MPMAC}}{\chi_{4, i} \mid X^{q}}\right) .
\end{aligned}
$$

Now we analyze each term in Eq. (8). For this analysis, for some $i \neq j$, we fix the $i$-th and $j$-th queries to $x_{i}=\left(x_{i[1]}, \ldots, x_{i[m]}\right)$ and $x_{j}=\left(x_{j[1]}, \ldots, x_{j\left[m^{\prime}\right]}\right)$ with $x_{i} \neq x_{j}$. We start with the first term. Collision $S_{i}=S_{j}$ is equivalent to

$$
V_{i} \oplus V_{j} \oplus 2^{m} 3^{\mathrm{I}\left(x_{i}\right)} L \oplus 2^{m^{\prime}} 3^{\mathrm{I}\left(x_{j}\right)} L=0 .
$$

To prove the maximum probability of Eq. (9), we need to use a case analysis.

Case 1: $m=m^{\prime}=1$. In this case, $V_{i} \oplus V_{j}=\operatorname{pad}\left(x_{i}\right) \oplus \operatorname{pad}\left(x_{j}\right)$. If $\mathrm{I}\left(x_{i}\right) \neq \mathrm{I}\left(x_{j}\right)$, the L.H.S. of Eq. (9) is a permutation of $L$ from Lemma 1. Thus, the probability of Eq. (9) is $1 / 2^{n}$. If $\mathrm{I}\left(x_{i}\right)=\mathrm{I}\left(x_{j}\right)$, the probability is zero as $\operatorname{pad}\left(x_{i}\right) \neq \operatorname{pad}\left(x_{j}\right)$. Case 2: $m>1, m^{\prime}=1$. In this case, the probability of Eq. (9) is obviously at most $(m-1) / 2^{n}+1 /\left(2^{n}-(m-1)\right) \leq(\ell-1) / 2^{n}+1 /\left(2^{n}-(\ell-1)\right)$ from the second claim of Lemma 2

Case 3: $m=m^{\prime}>1$. If the first $m-1$ blocks of $x_{i}$ and $x_{j}$ are the same, the probability is at most $1 / 2^{n}$, which is the same as in Case 1. Otherwise, Eq. (9) 
occurs with a probability of at most $(2 \ell-2) / 2^{n}+1 /\left(2^{n}-(2 \ell-2)\right)$ from the first claim of Lemma 2 .

Case 4: $m>1, m^{\prime}>1, m \neq m^{\prime}$. The bound of Case 3 also holds true.

Thus, we have

$$
\max _{X^{q}} P_{\overline{\chi_{1}} \mid X^{q}}^{\mathrm{MPMAC}} \leq \sum_{i<j} \max _{X^{q}} P_{\left[S_{i}=S_{j}\right] \mid X^{q}}^{\mathrm{MPMAC}} \leq\left(\begin{array}{l}
q \\
2
\end{array}\right)\left(\frac{2 \ell-2}{2^{n}}+\frac{1}{2^{n}-(2 \ell-2)}\right)
$$

For the second term, observe that $\overline{\chi_{2, i}}$ is the logical sum of events such that $S_{i}=2^{h} L \oplus x_{i^{\prime}[h]}$ for some $i^{\prime}$ including $i$, and $1 \leq h \leq \ell-1$. As $x_{i}$ has $m$ blocks, this is equivalent to $V_{i}=x_{i^{\prime}[h]} \oplus 2^{m} 3^{\mathrm{I}\left(x_{i}\right)} L \oplus 2^{h} L$. From Lemma 1], we have $2^{m} 3^{\mathrm{I}\left(x_{i}\right)} \neq$ $2^{h}$. Thus, it is enough to evaluate the maximum of $\operatorname{Pr}\left[V_{i}=\mathrm{u}_{1} L \oplus \mathrm{u}_{2} \mid \chi_{4, i}\right]$ for all $\mathrm{u}_{1} \in \Sigma^{n} \backslash\{0\}, \mathrm{u}_{2} \in \Sigma^{n}$. We fix $\mathrm{u}_{1} \neq 0$ and $\mathrm{u}_{2}$, and let $f(z)=\mathrm{u}_{1} z \oplus \mathrm{u}_{2}$ and $\mathrm{u}_{3} \stackrel{\text { def }}{=}\left(\mathrm{u}_{2} \oplus \operatorname{pad}\left(x_{i[m]}\right)\right) / \mathrm{u}_{1}$, where / denotes field division. We assume that $m>1$ and $L=\mathrm{u}_{3}$ satisfies $\chi_{4, i}$ with $x_{i}$. Note that $\operatorname{Pr}\left[V_{i}=f(L) \mid \chi_{4, i}\right]$ equals:

$$
\begin{aligned}
& \sum_{c} \operatorname{Pr}\left[V_{i}=f(L) \mid L=c\right] \operatorname{Pr}\left[L=c \mid \chi_{4, i}\right]+\operatorname{Pr}\left[V_{i}=0 \mid L=\mathrm{u}_{3}\right] \operatorname{Pr}\left[L=\mathrm{u}_{3} \mid \chi_{4, i}\right] \\
& \leq \max _{c} \operatorname{Pr}\left[V_{i}=f(c) \mid L=c\right]+\operatorname{Pr}\left[L=\mathrm{u}_{3} \mid \chi_{4, i}\right] \\
& =\max _{c} \operatorname{Pr}\left[\operatorname{Sum}(c)=f(c) \oplus \operatorname{pad}\left(x_{i[m]}\right) \mid L=c\right]+\operatorname{Pr}\left[L=\mathrm{u}_{3} \mid \chi_{4, i}\right]
\end{aligned}
$$

where the sum and maximums are taken for all $c \neq \mathrm{u}_{3}$ that satisfies $\chi_{4, i}$, and $\operatorname{Sum}(c)=\mathrm{P}_{n}\left(x_{i[1]} \oplus 2 c\right) \oplus \ldots \oplus \mathrm{P}_{n}\left(x_{i[m-1]} \oplus 2^{m-1} c\right)$. If every element in $\left\{\left(x_{i[1]} \oplus\right.\right.$ $\left.2 c), \ldots,\left(x_{i[m-1]} \oplus 2^{m-1} c\right)\right\}$ is in an even equivalent set, $\operatorname{Sum}(c)$ is 0 while $f(c) \oplus$ $\operatorname{pad}\left(x_{i[m]}\right) \neq 0$ from $c \neq \mathrm{u}_{3}$. If there exists any element which is in an odd equivalent set, $\operatorname{Sum}(c)$ is the sum of $k$ URP outputs for distinct inputs, for some $1 \leq k \leq m-1$. These inputs are not 0 as $c$ satisfies $\chi_{4, i}$. Therefore, the first term of the R.H.S. of Eq. (12) is at most $1 /\left(2^{n}-(\ell-1)\right)$ from Eq. (44). Also, the second term of the R.H.S. of Eq. (12) is at most $1 /\left(2^{n}-(\ell-1)\right)$. From these observations, $\max _{\mathrm{u}_{1} \neq 0, \mathrm{u}_{2}} \operatorname{Pr}\left[V_{i}=\mathrm{u}_{1} L \oplus \mathrm{u}_{2} \mid \chi_{4, i}\right]$ is at most $2 /\left(2^{n}-(\ell-1)\right)$ if $m>1$ and $L=\mathrm{u}_{3}$ satisfies $\chi_{4, i}$. For other cases (i.e., when $m=1$ or $m>1$ and $L=\mathrm{u}_{3}$ does not satisfy $\left.\chi_{4, i}\right)$, this bound also holds true. Therefore,

$$
\max _{X^{q}} P_{\overline{\chi_{2, i}} \mid \chi_{4, i}, X^{q}}^{\mathrm{MPMAC}} \leq(\ell-1) q \cdot \max _{\mathrm{u}_{1} \neq 0, \mathrm{u}_{2}} \operatorname{Pr}\left[V_{i}=\mathrm{u}_{1} L \oplus \mathrm{u}_{2} \mid \chi_{4, i}\right]=\frac{2(\ell-1) q}{2^{n}-(\ell-1)}
$$

holds for any $1 \leq i \leq q$, where the inequality holds since $\mathbf{M}^{(q)}$ contains at most $(\ell-1) q$ distinct elements. For the third and fourth terms of Eq. (8), we have

$$
\max _{X^{q}} P \frac{2}{\mathrm{MPMAC}_{\chi_{3, i}} \mid \chi_{4, i}, X^{q}} \leq \frac{2}{2^{n}-(\ell-1)}, \text { and } \max _{X^{q}} P_{\overline{\chi^{4, i}} \mid X^{q}}^{\mathrm{MPMAC}} \leq \frac{(\ell-1)}{2^{n}},
$$

where the first inequality follows from the same analysis as for the second term, and the second inequality holds since $\overline{\chi_{4, i}}$ occurs if $L$ takes one of (at most) $\ell-1$ 
values defined by $x_{i}$. Combining Eqs. (10), (13), and (14), we get

$$
\begin{aligned}
\max _{X^{q}} P_{\overline{a_{q}} \mid X^{q}}^{\mathrm{MPMAC}} & \leq\left(\begin{array}{l}
q \\
2
\end{array}\right)\left(\frac{2 \ell-2}{2^{n}}+\frac{1}{2^{n}-(2 \ell-2)}\right)+\frac{2(\ell-1) q^{2}+2 q}{2^{n}-(\ell-1)}+\frac{(\ell-1) q}{2^{n}} \\
& \leq \frac{1}{2^{n}-(2 \ell-2)}\left((3 \ell-2.5) q^{2}+1.5 q\right) .
\end{aligned}
$$

Note that $a_{q}$ implies $V_{i} \neq V_{j}$ if $i$-th and $j$-th tweaks are the same, for all $1 \leq i<j \leq q$. Therefore, if $a_{q}$ is given, the collision probability between $Y_{i}$ and $Y_{j}$ is at most $1 / 2^{n}$ for all fixed $q$ queries. Thus we have

$$
\max _{X^{q}} P \frac{\mathrm{MPMAC}}{\bar{b}_{q} \mid a_{q} X^{q}} \leq \sum_{i<j} \max _{X^{q}} P_{\left[Y_{i}=Y_{j}\right] \mid a_{q} X^{q}}^{\mathrm{MPMAC}} \leq\left(\begin{array}{l}
q \\
2
\end{array}\right) \frac{1}{2^{n}} .
$$

ANALYSIS FOR $\nu_{\ell}\left(\mathrm{MPMAC}, \overline{d_{q} \wedge e_{q}}\right)$. We consider a tweakable function, $G$, having $n$-bit input and output and tweak space $\mathcal{T}=\mathbb{I} \times \mathbb{J}$, where $\mathbb{I}=\left\{1, . ., 2^{n / 2}\right\}$ and $\mathbb{J}=\{0,1,2\}$. For any input $x \in \Sigma^{n}$ and tweak $t=\left(t_{[1]}, t_{[2]}\right) \in \mathcal{T}$, it is defined as $G(t, x) \stackrel{\text { def }}{=} \mathrm{P}_{n}(x)$ if $t_{[2]}=0$, otherwise $G(t, x) \stackrel{\text { def }}{=} \widetilde{\mathrm{P}}_{n}\left(\left(t_{[1]}, t_{[2]}\right), x\right)$, where $\mathrm{P}_{n}$ and $\widetilde{\mathrm{P}}_{n}$ are independent. If we allow an adversary against $G$ to make $(\ell-1) q$ queries for $\mathrm{P}_{n}$ and $q$ queries for $\widetilde{\mathrm{P}}_{n}$ (the order of query is arbitrary), he can simulate any $(q, \ell)$-CPA against MPMAC. Here, we assume that $L=\mathrm{P}_{n}(0)$ is publicly available, so that $\ell q$ queries are enough to simulate an attack. Moreover, if a $G$ based simulation generates distinct $\ell q$ outputs of $G$, this implies the occurrence of $d_{q}$ in MPMAC 3 . From these observations, $\nu_{\ell}\left(\mathrm{MPMAC}, \overline{d_{q}}\right)$ is at most

$$
\nu_{\tilde{\ell q}}\left(G, \overline{\left.\operatorname{dist}\left(\mathbf{Y}^{(\ell q)}\right)\right)}=\mu_{\widetilde{\ell q}}\left(G, \overline{\operatorname{dist}\left(\mathbf{Y}^{(\ell q)}\right)}\right) \leq \frac{(\ell-1) q^{2}}{2^{n}}+\left(\begin{array}{l}
q \\
2
\end{array}\right) \frac{1}{2^{n}},\right.
$$

where $\mathbf{Y}^{(\ell q)}$ is the set of $\ell q$ outputs, and $\widetilde{\ell q}$ means that the adversary can make $(\ell-1) q$ queries for $\mathrm{P}_{n}$ and $q$ queries for $\widetilde{\mathrm{P}}_{n}$, and the equality follows from an analysis similar to the one used for the proof of Lemma 4, The last inequality is trivial. Similarly, we can prove $\nu_{\ell}\left(\mathrm{MPMAC}, \overline{e_{q}}\right) \leq q / 2^{n}$ using $G$. Thus we have

$$
\nu_{\ell}\left(\mathrm{MPMAC}, \overline{d_{q} \wedge e_{q}}\right) \leq \frac{(\ell-1) q^{2}}{2^{n}}+\left(\begin{array}{l}
q \\
2
\end{array}\right) \frac{1}{2^{n}}+\frac{q}{2^{n}}
$$

using Lemma 9. Combining Eqs. (15), (16), and (17) and Lemma 4, Lemma 3 is proved.

Proving Theorem 2, Deriving an upper bound of $\operatorname{Adv}_{\text {MPMAC }}^{\text {vilquf }}(q, \ell)$ is easy since MPMAC can be seen as an instance of the Carter-Wegman MAC 20] (CW$\mathrm{MAC}$ ). Since the following lemma is almost the same as previous CW-MAC lemmas (e.g., Lemma 4 of [5]), we omit the proof here.

${ }^{3}$ We assume that the adversary never makes colliding queries and a pair of queries such as $\left(\left(t_{[1]}, t_{[2]}\right), x\right)$ and $\left(\left(t_{[1]}^{\prime}, t_{[2]}^{\prime}\right), x^{\prime}\right)$ with $t_{[2]}=t_{[2]}^{\prime}=0, x=x^{\prime}$, and $t_{[1]} \neq t_{[1]}^{\prime}$. These queries are obviously useless for simulation. 
Lemma 5. $\operatorname{Adv}_{\text {MPMAC }}^{\text {vilqrf }}(q, \ell) \leq\left(\begin{array}{l}q \\ 2\end{array}\right) \operatorname{dp}(\ell-1)+\left(\begin{array}{l}q \\ 2\end{array}\right) / 2^{n}$, where $\operatorname{dp}(m)$ denotes $\max _{x, x^{\prime} \in\left(\Sigma^{n}\right) \leq m, x \neq x^{\prime}, \mathrm{u} \in \Sigma^{n}} \operatorname{Pr}\left[\operatorname{PHASH}(x) \oplus \operatorname{PHASH}\left(x^{\prime}\right)=\mathrm{u}\right]$.

Finally, combining Lemmas 2, 3] and 5] we obtain

$$
\begin{aligned}
\operatorname{Adv}_{\operatorname{PMAC}\left[\mathrm{P}_{n}\right]}^{\mathrm{vilquf}}(q, \ell) & \leq \operatorname{Adv}_{\mathrm{PMAC}\left[\mathrm{P}_{n}\right], \operatorname{MPMAC}}^{\mathrm{cpa}}(q, \ell)+\operatorname{Adv}_{\mathrm{MPMAC}}^{\mathrm{vilqrf}}(q, \ell), \text { and } \\
& \leq \frac{(4 \ell-2.5) q^{2}+1.5 q}{2^{n}-2 \ell}+\left(\begin{array}{l}
q \\
2
\end{array}\right)\left(\frac{2 \ell-2}{2^{n}}+\frac{1}{2^{n}-(2 \ell-2)}+\frac{1}{2^{n}}\right) \\
& \leq \frac{(5 \ell-2.5) q^{2}+(1.5-\ell) q}{2^{n}-2 \ell} \leq \frac{5 \ell q^{2}}{2^{n}-2 \ell},
\end{aligned}
$$

where the last inequality holds since $q, \ell \geq 1$. This concludes the proof of Theorem 2 .

\section{TMAC and XCBC}

\subsection{New Security Bounds for TMAC and XCBC}

Since CBC-MAC provides no security if two messages with the same prefix are processed, a number of modifications have been proposed to make CBC-MAC secure for any message. EMAC, an early attempt, uses two blockcipher keys; TMAC [13] and XCBC [7] were later proposed as better solutions: they use one blockcipher key and some additional keys, and thus avoid two blockcipher key schedulings. TMAC and XCBC are defined as follows. Let CBC be the CBC-MAC function using $\mathrm{P}_{n}$; that is, for input $x=\left(x_{[1]}, \ldots, x_{[m]}\right) \in\left(\Sigma^{n}\right)^{m}$, $\mathrm{CBC}(x)=C_{m}$, where $C_{i}=\mathrm{P}_{n}\left(x_{[i]} \oplus C_{i-1}\right)$ and $C_{0}=0$. Let TMAC $\left[\mathrm{P}_{n}\right]$ denote the TMAC using $\mathrm{P}_{n}$. For input $x \in \Sigma^{*}$, TMAC $\left[\mathrm{P}_{n}\right]$ works as follows. First, we partition $x$ into $x=\left(x_{[1]}, \ldots, x_{[m]}\right)$, where $m=\|x\|_{n}$. If $m>1$, the tag for $x$ is $Y=\mathrm{P}_{n}\left(\mathrm{CBC}(\widehat{x}) \oplus \operatorname{pad}\left(x_{[m]}\right) \oplus 2^{\mathrm{I}(x)-1} L\right)$, where $\widehat{x}=\left(x_{[1]}, \ldots, x_{[m-1]}\right)$ and $L$ is independent and uniform over $\Sigma^{n}$. If $m=1, Y$ is $\mathrm{P}_{n}\left(\operatorname{pad}(x) \oplus 2^{\mathrm{I}(x)-1} L\right)$. Note that the $\mathrm{P}_{n}$ used in $\mathrm{CBC}$ and the one used in the finalization are identical. Therefore, in practice, TMAC has one blockcipher key and an additional $n$-bit key $L$. XCBC is similar to TMAC, but uses two $n$-bit keys, $L_{1}$ and $L_{2}$, as masking values instead of $L$ and $2 L$. The previous bound of TMAC $\left[\mathrm{P}_{n}\right]$ is $\left(3 \ell^{2}+1\right) q^{2} / 2^{n}\left[13\right.$ against $(q, \ell)-\mathrm{CPA}$, and $3 \sigma^{2} / 2^{n}$ against $(q, \sigma)$-CPA [10]. Almost the same results are obtained for XCBC [1013. However, using our proof approach in Sect. 3 and Bellare et al.'s analysis of the CBC function [5], we obtain the following.

Theorem 3. Let TMAC $\left[\mathrm{P}_{n}\right]$ be the TMAC using $\mathrm{P}_{n}$. We then have

$$
\operatorname{Adv}_{\operatorname{TMAC}\left[\mathrm{P}_{n}\right]}^{\operatorname{vilqrf}}(q, \ell) \leq \frac{4 \ell q^{2}}{2^{n}}+\frac{64 \ell^{4} q^{2}}{2^{2 n}}
$$

The proof of Theorem 3 is in the next section. The bound of Theorem 3 is also applicable to XCBC. The proof for XCBC is the same as the proof of Theorem 3 , thus we omit it here. 


\subsection{Proof of Theorem 3}

Since the proof structure is the same as that of Theorem 2, we give only a sketch of the proof. We define a modified TMAC, denoted by MTMAC, that uses an independent tweakable URP for its finalization. In MTMAC, we partition message $x$ into $\left(x_{[1]}, \ldots, x_{[m]}\right)$, where $m=\|x\|_{n}$, and when $m>1$, the tag is $Y=\widetilde{\mathrm{P}}_{n}\left(\mathrm{I}(x), \operatorname{CBC}(\widehat{x}) \oplus \operatorname{pad}\left(x_{[m]}\right)\right)$, where $\mathrm{I}(x) \in\{1,2\}$ is a tweak. When $m=1, Y=\widetilde{\mathrm{P}}_{n}(\mathrm{I}(x), \operatorname{pad}(x))$. For both TMAC $\left[\mathrm{P}_{n}\right]$ and MTMAC, let $X_{i} \in \Sigma^{*}$ be the $i$-th query and $\mathbf{M}^{(q)}\left(\mathbf{C}^{(q)}\right)$ be the set of inputs (outputs) to $\mathrm{P}_{n}$ generated in the CBC function for all $q$ queries. We also define $Y_{i}$ as the $i$-th tag, and $\mathbf{Y}^{(q)} \stackrel{\text { def }}{=}\left\{Y_{i}\right\}_{i=1, \ldots, q}$. When $\left\|X_{i}\right\|_{n}=m>1$, we define $V_{i}$ as the XOR of the $i$-th CBC output and $\operatorname{pad}\left(X_{i[m]}\right)$, and when $m=1, V_{i}=\operatorname{pad}\left(X_{i}\right)$. Moreover, $S_{i} \stackrel{\text { def }}{=} V_{i} \oplus 2^{\mathrm{I}\left(X_{i}\right)-1} L$, and $\mathbf{S}^{(q)} \stackrel{\text { def }}{=}\left\{S_{i}\right\}_{i=1, \ldots, q}$. In MTMAC, $S_{i}$ is a dummy variable. Note that $Y_{i}=\mathrm{P}_{n}\left(S_{i}\right)$ in TMAC $\left[\mathrm{P}_{n}\right]$ and that $Y_{i}=\widetilde{\mathrm{P}}_{n}\left(\mathrm{I}\left(X_{i}\right), V_{i}\right)$ in MTMAC, where $m=\left\|X_{i}\right\|_{n}$. We define $a_{q} \stackrel{\text { def }}{=} \operatorname{dist}\left(\mathbf{S}^{(q)}\right) \wedge\left[\mathbf{M}^{(q)} \cap \mathbf{S}^{(q)}=\emptyset\right]$ and $b_{q} \stackrel{\text { def }}{=} \operatorname{dist}\left(\mathbf{Y}^{(q)}\right), d_{q} \stackrel{\text { def }}{=}\left[\mathbf{C}^{(q)} \cap \mathbf{Y}^{(q)}=\emptyset\right]$. We then obtain

$$
\operatorname{Adv}_{\operatorname{TMAC}\left[\mathrm{P}_{n}\right], \operatorname{MTMAC}}^{\mathrm{cpa}}(q, \ell) \leq \nu_{\ell}\left(\operatorname{MTMAC}, \overline{a_{q} \wedge b_{q}}\right)+\nu_{\ell}\left(\operatorname{MTMAC}, \overline{d_{q}}\right)
$$

for any $(q, \ell)$ using an argument similar to that used for Lemma 3. Note that $a_{q}$ does not contain $\left[0 \notin \mathbf{M}^{(q)} \cup \mathbf{S}^{(q)}\right]$, as we do not have to care about 0 being an input to $\mathrm{P}_{n}$. Since Lemma 4 does not depend on the structure of message-hashing part, it also applies to MTMAC and we have

$$
\nu_{\ell}\left(\operatorname{MTMAC}, \overline{a_{q} \wedge b_{q}}\right)=\mu_{\ell}\left(\operatorname{MTMAC}, \overline{a_{q} \wedge b_{q}}\right) \leq \max _{X^{q}} P_{\overline{a_{q}} \mid X^{q}}^{\mathrm{MTMAC}}+\max _{X^{q}} P_{\overline{b_{q}} \mid a_{q} X^{q}}^{\mathrm{MTMAC}} .
$$

To obtain bounds of last two terms of Eq. (21), we need the following lemmat. It generalizes a lemma of Bellare et al. [5].

\section{Lemma 6.}

$$
\begin{gathered}
\max _{x \in\left(\Sigma^{n}\right)^{m}, x^{\prime} \in\left(\Sigma^{n}\right)^{m^{\prime}}, x \neq x^{\prime}, \mathrm{u} \in \Sigma^{n}} \operatorname{Pr}\left[\mathrm{CBC}(x) \oplus \mathrm{CBC}\left(x^{\prime}\right)=\mathrm{u}\right] \leq \frac{2 \mathrm{~d}\left(m^{*}\right)}{2^{n}}+\frac{64\left(m^{*}\right)^{4}}{2^{2 n}}, \\
\max _{x \in\left(\Sigma^{n}\right)^{m}, \mathrm{u} \in \Sigma^{n}} \operatorname{Pr}[\mathrm{CBC}(x)=\mathrm{u}] \leq \frac{2 \mathrm{~d}(m+1)}{2^{n}}+\frac{64(m+1)^{4}}{2^{2 n}},
\end{gathered}
$$

where $\mathrm{d}(m)$ is the maximum number of positive integers that divide $h$, for all $h \leq m$, and $m^{*}=\max \left\{m, m^{\prime}\right\}+1$.

Proof. (of Lemma 6) For any $z \in \Sigma^{n}, \mathrm{CBC}(x) \oplus \mathrm{CBC}\left(x^{\prime}\right)=\mathrm{u}$ is equivalent to $\mathrm{P}_{n}(\mathrm{CBC}(x) \oplus z)=\mathrm{P}_{n}\left(\mathrm{CBC}\left(x^{\prime}\right) \oplus z \oplus \mathrm{u}\right)$, which is equivalent to $\mathrm{CBC}(x \| z)=$ $\operatorname{CBC}\left(x^{\prime} \|(z \oplus \mathrm{u})\right)$. From Lemma 5 of [5], we see that the collision probability of

${ }^{4}$ Pietrzak [18] proved that the collision probability of CBC among $q$ messages could be smaller than the union bound applied to Lemma 6 for some $(q, \ell)$. Since our analysis is based on the union bound, we do not know if the result of [18] can be combined into our proof to obtain other proofs. 
$\mathrm{CBC}(x \| z)$ and $\operatorname{CBC}\left(x^{\prime} \|(z \oplus \mathrm{u})\right)$ is at most $2 \mathrm{~d}\left(m^{*}\right) / 2^{n}+64\left(m^{*}\right)^{4} / 2^{2 n}$ for any $z$ (note that $x\left\|z \neq x^{\prime}\right\|\left(z \oplus \mathrm{u}\right.$ ) holds for any $z$ and $\mathrm{u}$ as we assumed $\left.x \neq x^{\prime}\right)$. Therefore, the first claim is proved. The second can be similarly proved.

We analyze $\max _{X^{q}} P_{\overline{a_{q}} \mid X^{q}}^{\mathrm{MTMAC}}$. If the $i$-th and $j$-th queries are fixed to $x_{i}$ and $x_{j}$ with $x_{i} \neq x_{j}$, collision $S_{i}=S_{j}$ is equivalent to $V_{i} \oplus 2^{\mathrm{I}\left(x_{i}\right)} L=V_{j} \oplus 2^{\mathrm{I}\left(x_{j}\right)} L$. If $\mathrm{I}\left(x_{i}\right) \neq \mathrm{I}\left(x_{j}\right)$, the collision occurs with probability $1 / 2^{n}$ since $L$ is independent of $V_{i}$ and $V_{j}$ and $2^{\mathrm{I}\left(x_{i}\right)} L \oplus 2^{\mathrm{I}\left(x_{j}\right)} L$ is a permutation of $L$ from Lemma 1 , If $\mathrm{I}\left(x_{i}\right)=\mathrm{I}\left(x_{j}\right), S_{i}=S_{j}$ implies $V_{i}=V_{j}$, which has a probability of at most $2 \mathrm{~d}(\ell) / 2^{n}+64 \ell^{4} / 2^{2 n}$ from Lemma 6 and a case analysis similar to the one used to derive Eq. (10). Therefore, the probability of $\overline{\operatorname{dist}\left(\mathbf{S}^{(q)}\right)}$ is at most $\left(\begin{array}{l}q \\ 2\end{array}\right)\left(2 \mathrm{~d}(\ell) / 2^{n}+\right.$ $\left.64 \ell^{4} / 2^{2 n}\right)$. Note that any collision event consisting of $\overline{\left[\mathbf{M}^{(q)} \cap \mathbf{S}^{(q)}=\emptyset\right]}$ has probability $1 / 2^{n}$ since $L$ is independent of all members of $\mathbf{M}^{(q)}$. From these observations, we have

$$
\begin{aligned}
& \max _{X^{q}} P \frac{\mathrm{MTMAC}}{\bar{a}_{q} \mid X^{q}} \leq \max _{X^{q}} P \frac{\mathrm{MTMAC}}{\operatorname{dist}\left(\mathbf{S}^{(q)}\right) \mid X^{q}}+\max _{X^{q}} P \frac{\mathrm{MTMAC}}{\left[\mathbf{M}^{(q)} \cap \mathbf{S}^{(q)}=\emptyset\right]} \mid X^{q} \\
& \leq\left(\begin{array}{l}
q \\
2
\end{array}\right)\left(\frac{2 \mathrm{~d}(\ell)}{2^{n}}+\frac{64 \ell^{4}}{2^{2 n}}\right)+\frac{(\ell-1) q^{2}}{2^{n}} .
\end{aligned}
$$

The analyses of $\max _{X^{q}} P \frac{\mathrm{MTMAC}}{b_{q} \mid a_{q} X^{q}}$ and $\nu_{\ell}\left(\mathrm{MTMAC}, \overline{d_{q}}\right)$ are the same as those used for the proof of Lemma 3 . We obtain

$$
\max _{X^{q}} P \overline{b_{q} \mid a_{q} X^{q}} \leq\left(\begin{array}{l}
q \\
2
\end{array}\right) \frac{1}{2^{n}}, \text { and } \nu_{\ell}\left(\operatorname{MTMAC}, \overline{d_{q}}\right) \leq \frac{(\ell-1) q^{2}}{2^{n}}+\left(\begin{array}{l}
q \\
2
\end{array}\right) \frac{1}{2^{n}} .
$$

As with MPMAC, MTMAC is an instance of CW-MAC. Thus, we have

$$
\operatorname{Adv}_{\text {MTMAC }}^{\text {vilqrf }}(q, \ell) \leq\left(\begin{array}{l}
q \\
2
\end{array}\right)\left(\frac{2 \mathrm{~d}(\ell)}{2^{n}}+\frac{64 \ell^{4}}{2^{2 n}}+\frac{1}{2^{n}}\right)
$$

Combining the bound of $\operatorname{Adv}_{\mathrm{TMAC}\left[\mathrm{P}_{n}\right], \operatorname{MTMAC}}^{\mathrm{cpa}}(q, \ell)$, which can be derived from Eqs. (20), (21), (22), and (23), and the bound of $\operatorname{Adv}_{\text {MTMAC }}^{\text {vilqrf }}(q, \ell)$ by Eq. (24), $\operatorname{Adv}_{\text {TMAC }^{\text {vilqrf }} \text { ] }}(q, \ell)$ is at most $\left((2 \mathrm{~d}(\ell)+2 \ell) q^{2}\right) / 2^{n}+64 \ell^{4} q^{2} / 2^{2 n}$. Since $\mathrm{d}(\ell) \leq \ell$, this concludes the proof of Theorem 3 .

\section{Conclusion and Future Work}

In this paper, we have provided new security bounds for PMAC, TMAC, and $\mathrm{XCBC}$. Our result demonstrates that the security degradation with respect to the maximum length of a message is linear for PMAC and almost linear (unless message is impractically long) for TMAC and XCBC, while previous analyses of these modes proved quadratic security degradation.

A Comparison of Bounds. As we mentioned, our new bounds improve the old ones under most (but not all) cases. Here, we give a detailed comparison between 
new and old bounds. For simplicity, we ignore the constants. Thus, the new PMAC bound is $\ell q^{2} / 2^{n}$, the new TMAC (and XCBC) bound is $\ell q^{2} / 2^{n}+\ell^{4} q^{2} / 2^{2 n}$, and the old bounds are $\sigma^{2} / 2^{n}$ for all. For PMAC, the new bound is better if and only if $\sqrt{\ell} q<\sigma$, i.e., the mean message block length $(\sigma / q)$ is larger than $\sqrt{\ell}$. Similarly, for TMAC and XCBC, the new bound is better if and only if the mean message block length is larger than $\sqrt{\ell(1+c)}$, where $c=\ell^{3} / 2^{n}$, which can be small in practice. Thus, the criterion for choosing a bound is the distance between the mean block length and the square root of the maximum block length.

As a concrete example, let $n=128, q=2^{40}$, and $\ell=2^{16}$. Then the new PMAC bound is $2^{-32}$ (the new TMAC and XCBC bounds are almost $2^{-32}$ ), while the old bound ranges from $2^{-48}$ to $2^{-16}$. The old bound is better if $99.9 \%$ of the messages are one-block, as $\sigma^{2} / 2^{n} \leq(1 \cdot 0.999 q+\ell \cdot 0.001 q)^{2} / 2^{n}<2^{-35}$. In this case, the mean block length is smaller than $2^{6}$, which is smaller than $\sqrt{\ell}=2^{8}$. In contrast, if $1 \%$ of the messages are $\ell$-block, the new bound is better since $\sigma^{2} / 2^{n} \geq(\ell \cdot 0.01 q+1 \cdot 0.99 q)^{2} / 2^{n}>2^{-30}$ and the mean block length is at least $2^{9}$. Generally, the new bounds are better when only a tiny fraction of the message length distribution is concentrated on the right.

On The Security of OMAC. OMAC [11, i.e., CMAC [1], is similar to TMAC, but uses a different finalization. In OMAC using $\mathrm{P}_{n}$, denoted by $\mathrm{OMAC}\left[\mathrm{P}_{n}\right], L$ is $\mathrm{P}_{n}(0)$, and, instead of using $2^{\mathrm{I}(x)-1} L$, it uses $2^{\mathrm{I}(x)} L$ as the masking value. Thus OMAC has only one blockcipher key. The known security bound of OMAC $\left[\mathrm{P}_{n}\right]$ is $\left(5 \ell^{2}+1\right) q^{2} / 2^{n}$ [1] against $(q, \ell)-\mathrm{CPA}$, and $4 \sigma^{2} / 2^{n}$ against $(q, \sigma)$-CPA [10. Unfortunately, we have not yet succeeded in showing new bounds. In a manner similar to that for TMAC, we define a modified 5 OMAC (MOMAC), using $\mathrm{P}_{n}$ and $\widetilde{\mathrm{P}}_{n}$, and define sets of variables, $\left(\mathbf{M}^{(q)}, \mathbf{C}^{(q)}, \mathbf{S}^{(q)}\right.$, and $\left.\mathbf{Y}^{(q)}\right)$, for both OMAC $\left[\mathbf{P}_{n}\right]$ and MOMAC. By defining events $a_{q} \stackrel{\text { def }}{=} \operatorname{dist}\left(\mathbf{S}^{(q)}\right) \wedge\left[\mathbf{M}^{(q)} \cap \mathbf{S}^{(q)}=\emptyset\right] \wedge\left[0 \notin \mathbf{S}^{(q)}\right]$, $b_{q} \stackrel{\text { def }}{=} \operatorname{dist}\left(\mathbf{Y}^{(q)}\right), d_{q} \stackrel{\text { def }}{=}\left[\mathbf{C}^{(q)} \cap \mathbf{Y}^{(q)}=\emptyset\right]$, and $e_{q} \stackrel{\text { def }}{=}\left[L \notin \mathbf{Y}^{(q)}\right]$, we can prove that $\operatorname{Adv}_{\left.\mathrm{OMAC}^{\mathrm{cpa}} n\right], \operatorname{MOMAC}}(q, \ell)$ is at most $\nu_{\ell}\left(\mathrm{MOMAC}, \overline{a_{q} \wedge b_{q} \wedge d_{q} \wedge e_{q}}\right)$. However, to obtain a bound of $\nu_{\ell}$ (MOMAC, $\overline{a_{q} \wedge b_{q}}$ ), we need the maximum probability of $\left[\mathrm{CBC}(x) \oplus \operatorname{CBC}\left(x^{\prime}\right)=\mathrm{u}_{1} L \oplus \mathrm{u}_{2}\right]$ for $\mathrm{u}_{1}=\left(2 \oplus 2^{2}\right)$, which corresponds to the sum of two distinct masking values, and for all $\mathrm{u}_{2}$, i.e., we need a generalization of Lemma 6. We think that this is an interesting open problem.

\section{Acknowledgments}

We would like to thank Tetsu Iwata and the anonymous referees for very useful comments and suggestions.

\section{References}

1. Recommendation for Block Cipher Modes of Operation: The CMAC Mode for Authentication. NIST Special Publication 800-38B, available from http://csrc.nist.gov/CryptoToolkit/modes/

5 The "modified OMAC" was also described in [11; however, our definition is different. 
2. den Boer, B., Boly, J.P., Bosselaers, A., Brandt, J., Chaum, D., Damgård, I., Dichtl, M., Fumy, W., van der Ham, M., Jansen, C.J.A., Landrock, P., Preneel, B., Roelofsen, G., de Rooij, P., Vandewalle, J.: RIPE Integrity Primitives, final report of RACE Integrity Primitives Evaluation (1995)

3. Bellare, M., Kilian, J., Rogaway, P.: The Security of the Cipher Block Chaining Message Authentication Code. Journal of Computer and System Science 61(3) (2000)

4. Bellare, M., Desai, A., Jokipii, E., Rogaway, P.: A Concrete Security Treatment of Symmetric Encryptiont. In: FOCS '97. Proceedings of the 38th Annual Symposium on Foundations of Computer Science, pp. 394-403 (1997)

5. Bellare, M., Pietrzak, K., Rogaway, P.: Improved Security Analyses for CBC MACs. In: Shoup, V. (ed.) CRYPTO 2005. LNCS, vol. 3621, pp. 527-541. Springer, Heidelberg (2005)

6. Bernstein, D.J.: Stronger Security Bounds for Wegman-Carter-Shoup Authenticators. In: Cramer, R.J.F. (ed.) EUROCRYPT 2005. LNCS, vol. 3494, pp. 164-180. Springer, Heidelberg (2005)

7. Black, J., Rogaway, P.: CBC MACs for Arbitrary-Length Messages: The Three-Key Constructions. In: Bellare, M. (ed.) CRYPTO 2000. LNCS, vol. 1880, pp. 197-215. Springer, Heidelberg (2000)

8. Carter, L., Wegman, M.: Universal Classes of Hash Functions. Journal of Computer and System Science 18, 143-154 (1979)

9. Goldreich, O.: Modern Cryptography, Probabilistic Proofs and Pseudorandomness. In: Algorithms and Combinatorics, vol. 17, Springer, Heidelberg (1998)

10. Iwata, T., Kurosawa, K.: Stronger Security Bounds for OMAC, TMAC, and XCBC. In: Johansson, T., Maitra, S. (eds.) INDOCRYPT 2003. LNCS, vol. 2904, pp. 402 415. Springer, Heidelberg (2003)

11. Iwata, T., Kurosawa, K.: OMAC: One-Key CBC MAC. In: Johansson, T. (ed.) FSE 2003. LNCS, vol. 2887, pp. 129-153. Springer, Heidelberg (2003)

12. Krovetz, T., Rogaway, P.: The OCB Authenticated-Encryption Algorithm. Internet Draft (2005)

13. Kurosawa, K., Iwata, T.: TMAC: Two-Key CBC MAC. In: Joye, M. (ed.) CT-RSA 2003. LNCS, vol. 2612, pp. 33-49. Springer, Heidelberg (2003)

14. Liskov, M., Rivest, R.L., Wagner, D.: Tweakable Block Ciphers. In: Yung, M. (ed.) CRYPTO 2002. LNCS, vol. 2442, pp. 31-46. Springer, Heidelberg (2002)

15. Maurer, U.: Indistinguishability of Random Systems. In: Knudsen, L.R. (ed.) EUROCRYPT 2002. LNCS, vol. 2332, pp. 110-132. Springer, Heidelberg (2002)

16. Maurer, U., Pietrzak, K.: Composition of Random Systems: When Two Weak Make One Strong. In: Naor, M. (ed.) TCC 2004. LNCS, vol. 2951, pp. 410-427. Springer, Heidelberg (2004)

17. Pietrzak, K.: Composition Does Not Imply Adaptive Security. In: Shoup, V. (ed.) CRYPTO 2005. LNCS, vol. 3621, pp. 55-65. Springer, Heidelberg (2005)

18. Pietrzak, K.: A Tight Bound for EMAC. In: Bugliesi, M., Preneel, B., Sassone, V., Wegener, I. (eds.) ICALP 2006. LNCS, vol. 4052, pp. 168-179. Springer, Heidelberg (2006)

19. Rogaway, P.: Efficient Instantiations of Tweakable Blockciphers and Refinements to Modes OCB and PMAC. In: Lee, P.J. (ed.) ASIACRYPT 2004. LNCS, vol. 3329, pp. 16-31. Springer, Heidelberg (2004), (September 24, 2006) http://www.cs.ucdavis.edu/ rogaway/papers/offsets.pdf

20. Wegman, M., Carter, L.: New Hash Functions and Their Use in Authentication and Set Equality. Journal of Computer and System Sciences 22, 265-279 (1981) 


\section{A Lemmas from Maurer's Methodology}

We describe some lemmas developed by Maurer (e.g., [15]) that we used. We assume that $F$ and $G$ are two random functions with the same input/output size; we define MESs $\mathcal{A}=a_{0} a_{1} \ldots$ and $\mathcal{B}=b_{0} b_{1} \ldots$ for $F$ and $G$. The $i$-th input and output are denoted by $X_{i}$ and $Y_{i}$ for $F$ (or $G$ ), respectively. Equality of (possibly conditional) probability distributions means equality as functions, i.e., equality holds for all possible arguments. For example, we write $P_{Y^{i} \mid X^{i} a_{i}}^{F}=P_{Y^{i} \mid X^{i} b_{i}}^{G}$ to mean $P_{Y^{i} \mid X^{i} a_{i}}^{F}\left(y^{i}, x^{i}\right)=P_{Y^{i} \mid X^{i} b_{i}}^{G}\left(y^{i}, x^{i}\right)$ for all $\left(x^{i}, y^{i}\right)$, where $P_{a_{i} \mid X^{i}}^{F}\left(x^{i}\right)$ and $P_{b_{i} \mid X^{i}}^{G}\left(x^{i}\right)$ are positive. Inequalities, such as $P_{Y^{i} \mid X^{i} a_{i}}^{F} \leq P_{Y^{i} \mid X^{i} b_{i}}^{G}$, are similarly defined.

Lemma 7. (A corollary from Theorem 1 (i), Lemma 1 (iv), and Lemma 4 (ii) of [15]) Let $\mathbb{F}$ be the function of $F$ or $G$ (i.e., $\mathbb{F}[F]$ is a function that internally invokes $F$, possibly several times, to process its inputs). Here, $\mathbb{F}$ can be probabilistic, and, if so, $\mathbb{F}$ is independent of $F$ or $G$. Suppose that $P_{Y_{i} \mid X^{i} Y^{i-1} a_{i}}^{F}=P_{Y_{i} \mid X^{i} Y^{i-1} b_{i}}^{G}$ and $P_{a_{i} \mid X^{i} Y^{i-1} a_{i-1}}^{F} \leq P_{b_{i} \mid X^{i} Y^{i-1} b_{i-1}}^{G}$ holds for $i \geq 1$. We then have

$$
\operatorname{Adv}_{F, G}^{\mathrm{cpa}}(q) \leq \nu\left(F, \overline{a_{q}}\right) \text {, and } \operatorname{Adv}_{\mathbb{F}[F], \mathbb{F}[G]}^{\mathrm{cpa}}(q) \leq \nu\left(\mathbb{F}[F], \overline{a_{q}^{*}}\right) .
$$

Here, $M E S \mathcal{A}^{*}=a_{0}^{*} a_{1}^{*} \ldots$ is defined such that $a_{i}^{*}$ denotes $\mathcal{A}$-event is satisfied for time period $i$. For example, if $\mathbb{F}[F]$ always invokes $F k$ times for any input, then $a_{i}^{*} \equiv a_{k i}$.

Lemma 8. (Theorem 2 of [15]) If $P_{a_{i} \mid X^{i} Y^{i-1} a_{i-1}}^{F}=P_{a_{i} \mid X^{i} a_{i-1}}^{F}$ holds for $i \geq 1$, the maximum probabilities of $\overline{a_{q}}$ for all adaptive and non-adaptive attacks are the same, i.e., $\nu\left(F, \overline{a_{q}}\right)=\mu\left(F, \overline{a_{q}}\right)$.

Lemma 9. (Lemma 6 (iii) of [15]) If $M E S s \mathcal{A}=a_{0} a_{1} \ldots$ and $\mathcal{B}=b_{0} b_{1} \ldots$ are defined for $F$, we have $\nu\left(F, \overline{a_{q} \wedge b_{q}}\right) \leq \nu\left(F, \overline{a_{q}}\right)+\nu\left(F, \overline{b_{q}}\right)$.

These lemmas are easily extended even if the adversary's parameter $\theta$ contains $\ell$ (or $\sigma$ ) in addition to $q$.

\section{B Proof of the First and Second Inequalities of Lemma 3}

We define two tweakable functions having $n$-bit input/output and tweak space $\mathcal{T}=\left\{1, . ., 2^{n / 2}\right\} \times\{0,1,2\}$. For any input $x \in \Sigma^{n}$ and tweak $t=\left(t_{[1]}, t_{[2]}\right) \in \mathcal{T}$,

$$
\begin{aligned}
& \mathrm{XE}(t, x) \stackrel{\text { def }}{=} \mathrm{P}_{n}\left(x \oplus 2^{t_{[1]}} 3^{t_{[2]}} L\right), \text { where } L=\mathrm{P}_{n}(0), \text { and } \\
& \widetilde{\mathrm{XE}}(t, x) \stackrel{\text { def }}{=} \begin{cases}\mathrm{P}_{n}\left(x \oplus 2^{t_{[1]}} 3^{t_{[2]}} L\right), & \text { if } t_{[2]}=0, \text { where } L=\mathrm{P}_{n}(0) ; \\
\widetilde{\mathrm{P}}_{n}\left(\left(t_{[1]}, t_{[2]}\right), x\right), & \text { otherwise. }\end{cases}
\end{aligned}
$$

In the definition of $\widetilde{\mathrm{XE}}, \mathrm{P}_{n}$ and $\widetilde{\mathrm{P}}_{n}$ are assumed to be independent. It is obvious that PMAC $\left[\mathrm{P}_{n}\right]$ and MPMAC can be realized by using XE and $\widetilde{\mathrm{XE}}$ in a black-box 
manner. We consider a game in which an adversary tries to distinguish XE from $\widetilde{\mathrm{XE}}$ using $q$ queries. Note that a query is in $\mathcal{T} \times \Sigma^{n}$. Let $\left(T_{i}, X_{i}\right) \in \mathcal{T} \times \Sigma^{n}$ be the $i$-th query, and $Y_{i} \in \Sigma^{n}$ be the $i$-th output. In addition, let $S_{i}$ be $X_{i} \oplus 2^{T_{i[1]}} 3^{T_{i[2]}} L$, where $T_{i}=\left(T_{i[1]}, T_{i[2]}\right)$. For $\widetilde{\mathrm{XE}}, S_{i}$ is defined as a dummy variable when $T_{i[2]} \neq 0$. We define the following two events:

$$
\begin{aligned}
a_{i}^{*} \stackrel{\text { def }}{=}\left[S_{j} \neq S_{k} \text { for all }(j, k) \in \xi(i)\right] \wedge\left[S_{j} \neq 0 \text { for all } j=1, \ldots, i\right] . \\
b_{i}^{*} \stackrel{\text { def }}{=}\left[Y_{j} \neq Y_{k} \text { for all }(j, k) \in \xi(i)\right] \wedge\left[Y_{j} \neq L \text { for all } j=1, \ldots, i\right], \\
\quad \text { where } \psi(i) \stackrel{\text { def }}{=}\left\{j: 1 \leq j \leq i, T_{j[2]} \in\{1,2\}\right\}, \text { and } \\
\quad \xi(i) \stackrel{\text { def }}{=}\left\{(j, k) \in\{1, \ldots, i\}^{2}: j \neq k, \text { at least one of } j \text { or } k \text { is in } \psi(i)\right\} .
\end{aligned}
$$

Note that $\psi(i)$ and $\xi(i)$ depend on $\left\{T_{j[2]}\right\}_{j=1, \ldots, i}$. Clearly, $\mathcal{A}^{*}=a_{0}^{*} a_{1}^{*} \ldots$ and $\mathcal{B}^{*}=b_{0}^{*} b_{1}^{*} \ldots$ are MESs and they are equivalent in XE (but not in $\widetilde{\mathrm{XE}}$ ). Also, note that $\mathcal{A}^{*}$ and $\mathcal{B}^{*}$ defined for XE $(\widetilde{\mathrm{XE}})$ are compatible with MESs defined for $\mathrm{PMAC}\left[\mathrm{P}_{n}\right]$ (MPMAC). For example, if one uses XE to simulate an attack against PMAC $\left[\mathrm{P}_{n}\right]$ and observes $a_{i}^{*}$ in time period $i, a_{i^{\prime}}$ is occurring for the $i^{\prime}$-th query to $\operatorname{PMAC}\left[\mathrm{P}_{n}\right]$, for some $i^{\prime} \leq i$. We then have

$$
P_{Y_{i} \mid X^{i} T^{i} Y^{i-1} a_{i}^{*}}^{\mathrm{XE}}=\sum P_{Y_{i} \mid L X^{i} T^{i} Y^{i-1}}^{\mathrm{XE}} \cdot P_{L \mid X^{i} T^{i} Y^{i-1} a_{i}^{*}}^{\mathrm{XE}},
$$

where the summation is taken for all $L \in \Gamma\left(x^{i}, t^{i}, y^{i-1}\right)$, which is the set of $L=c$ such that the rightmost term is non-zero. The equality of Eq. (25) holds since $S^{i}$ is completely determined if $X^{i}$ and $L$ are fixed.

We focus on the rightmost two terms of Eq. (25) for some fixed $X^{i}=x^{i}$, $T^{i}=t^{i}, Y^{i-1}=y^{i-1}$ satisfying $b_{i-1}^{*}$, and $L=c \in \Gamma\left(x^{i}, t^{i}, y^{i-1}\right)$ (thus $S^{i}=s^{i}$ is also fixed). It is clear that $P_{L \mid X^{i} T^{i} Y^{i-1} a_{i}^{*}}^{\mathrm{XE}}\left(c, x^{i}, t^{i}, y^{i-1}\right)$ is the uniform distribution over $\Gamma\left(x^{i}, t^{i}, y^{i-1}\right)$. The conditional probability $P_{Y_{i} \mid L X^{i} T^{i} Y^{i-1}}^{\mathrm{XE}}\left(y_{i}, c, x^{i}, t^{i}, y^{i-1}\right)$ is 1 if $y_{i}=y_{j}$, and $i \notin \psi(i)$ and $\exists j \notin \psi(i)$ such that $s_{i}=s_{j}$. If $i \in \psi(i)$ or $i \notin \psi(i)$ but $s_{i} \neq s_{j}$ for $j \leq i-1, Y_{i}$ is uniform over $\Sigma^{n} \backslash\left\{y_{1}, \ldots, y_{i-1}, c\right\}$.

Similarly, for $\widetilde{\mathrm{XE}}$, we have

$$
P_{Y_{i} \mid X^{i} T^{i} Y^{i-1}}^{\widetilde{\mathrm{XE}}} a_{i}^{*} b_{i}^{*}=\sum P_{Y_{i} \mid L X^{i} T^{i} Y^{i-1} b_{i}^{*}}^{\widetilde{\mathrm{XE}}} \cdot P_{L \mid X^{i} T^{i} Y^{i-1} a_{i}^{*} b_{i-1}^{*}}^{\widetilde{\mathrm{X}}},
$$

where the summation is taken for all $L \in \Gamma\left(x^{i}, t^{i}, y^{i-1}\right)$. Then, a simple case analysis shows that

$$
P_{Y_{i} \mid X^{i} T^{i} Y^{i-1} a_{i}^{*}}^{\mathrm{XE}}=P_{Y_{i} \mid X^{i} T^{i} Y^{i-1}}^{\widetilde{\mathrm{X}} a_{i}^{*} b_{i}^{*}} .
$$

Moreover, we have

$$
\begin{aligned}
& P_{a_{i}^{*} \mid X^{i} T^{i} Y^{i-1} a_{i-1}^{*}}^{\mathrm{XE}}=\sum P_{a_{i}^{*} \mid L X^{i} T^{i} Y^{i-1} a_{i-1}^{*}}^{\mathrm{XE}} \cdot P_{L \mid X^{i} T^{i} Y^{i-1} a_{i-1}^{*}}^{\mathrm{XE}}, \text { and } \\
& P_{a_{i}^{*} b_{i}^{*} \mid X^{i} T^{i} Y^{i-1} a_{i-1}^{*} b_{i-1}^{*}}=\sum P_{a_{i}^{*} b_{i}^{*} \mid L X^{i} T^{i} Y^{i-1} a_{i-1}^{*} b_{i-1}^{*}}^{\widetilde{\mathrm{XE}}} \cdot P_{L \mid X^{i} T^{i} Y^{i-1} a_{i-1}^{*} b_{i-1}^{*}},
\end{aligned}
$$


where the summations are taken for all $L \in \Gamma^{\prime}\left(x^{i-1}, t^{i-1}, y^{i-1}\right)$, which is the set of $L=c$ such that the last term of Eq. (28) (or Eq. (29)) is non-zero. It is easy to find that the last terms of Eqs. (28) and (29) are the same conditional distributions. However, we have $P_{a_{i}^{*} b_{i}^{*} \mid L X^{i} T^{i} Y^{i-1} a_{i-1}^{*} b_{i-1}^{*}} \leq P_{a_{i}^{*} \mid L X^{i} T^{i} Y^{i-1} a_{i-1}^{*}}^{\mathrm{XE}}$ since both sides are 0 if $L \notin \Gamma\left(x^{i}, t^{i}, y^{i-1}\right)$, and otherwise the R.H.S. is 1 . Thus we have

$$
P_{a_{i}^{*} b_{i}^{*} \mid X^{i} T^{i} Y^{i-1} a_{i-1}^{*} b_{i-1}^{*}}^{\widetilde{X_{0}^{*}}} \leq P_{a_{i}^{*} \mid X^{i} T^{i} Y^{i-1} a_{i-1}^{*}}^{\mathrm{XE}} .
$$

From Eqs. (27) and (30) and the second claim of Lemma 7, the first inequality of Lemma 3 is proved. The second follows from the first and Lemma 9.

\section{Proof of Lemma 4}

Note that $\mathbf{M}^{(i)}\left(\mathbf{C}^{(i)}\right)$ denotes the set of $\mathbf{P}_{n}$ inputs (outputs) generated in PHASH up to the $i$-th query. Let $\mathbf{Z}^{(i)}$ be the set of random variables $\left(L, \mathbf{C}^{(i)}\right)$. If $\mathbf{Z}^{(i)}$ and $X^{i}$ are fixed, $\mathbf{M}^{(i)}, V^{i}$, and $S^{i}$ are uniquely determined. We have

$$
\begin{aligned}
P_{a_{i} b_{i} \mid X^{i} Y^{i-1} a_{i-1} b_{i-1}}^{\mathrm{MPMC}}= & \sum_{\mathbf{Z}^{(i)}} P_{b_{i} \mid \mathbf{Z}^{(i)} X^{i} Y^{i-1} a_{i} b_{i-1}}^{\mathrm{MPMC}} \\
& \cdot P_{a_{i} \mid \mathbf{Z}^{(i)} X^{i} Y^{i-1} a_{i-1} b_{i-1}}^{\mathrm{MPMC}} \cdot P_{\mathbf{Z}^{(i)} \mid X^{i} Y^{i-1} a_{i-1} b_{i-1}}^{\mathrm{MPC}},
\end{aligned}
$$

where the summations are taken for all $\mathbf{Z}^{(i)}=\mathbf{z}^{(i)}$ such that $\left(\mathbf{z}^{(i)}, x^{i}\right)$ satisfies $a_{i-1}$. Note that $a_{i}$ implies that, if the $j$-th and $j^{\prime}$-th tweaks (recall that the $i$-th tweak is a function of $X_{i}$ ) are the same, $V_{j} \neq V_{j^{\prime}}$ holds for all $j, j^{\prime} \leq i$, $j \neq j^{\prime}$. From this, $P_{b_{i} \mid \mathbf{Z}^{(i)} X^{i} Y^{i-1} a_{i} b_{i-1}}^{\mathrm{MPMAC}}\left(\mathbf{z}^{(i)}, x^{i}, y^{i-1}\right)$ does not depend on $y^{i-1}$, and it is $\left(2^{n}-(i-1)\right) /\left(2^{n}-\pi\left(x^{i}\right)\right)$, where $\pi\left(x^{i}\right)$ is the number of indices $j \in\{1, \ldots, i-1\}$ such that the $j$-th and $i$-th tweaks are the same. Moreover, $P_{a_{i} \mid \mathbf{Z}^{(i)} X^{i} Y^{i-1} a_{i-1} b_{i-1}}^{\mathrm{MPMAC}}\left(\mathbf{z}^{(i)}, x^{i}, y^{i-1}\right)$ does not depend on $y^{i-1}$ as it is 1 if $\left(\mathbf{z}^{(i)}, x^{i}\right)$ satisfies $a_{i}$, and otherwise 0 . Finally, it is easy to see that $P_{\mathbf{Z}^{(i)} \mid X^{i} Y^{i-1} a_{i-1} b_{i-1}}^{\mathrm{MPMC}}$ equals $P_{\mathbf{Z}^{(i)} \mid X^{i} a_{i-1}}^{\mathrm{MPMAC}}$ (here, $b_{i-1}$ implies $V_{j} \neq V_{j^{\prime}}$ whenever $j$-th and $j^{\prime}$-th tweaks are the same, however, this is already implied by $\left.a_{i-1}\right)$. Thus, $P_{a_{i} b_{i} \mid X^{i} Y^{i-1} a_{i-1} b_{i-1}}^{\mathrm{MPMC}}$ does not depend on $y^{i-1}$, and, for any $x^{i}$ and $\widehat{y}^{i-1}$ satisfying $b_{i-1}$, we have

$$
\begin{aligned}
P_{a_{i} b_{i} \mid X^{i} a_{i-1} b_{i-1}}^{\mathrm{MPMAC}}\left(x^{i}\right) & =\sum P_{a_{i} b_{i} \mid X^{i} Y^{i-1} a_{i-1} b_{i-1}}^{\mathrm{MPMAC}}\left(x^{i}, y^{i-1}\right) \cdot P_{Y^{i-1} \mid X^{i} a_{i-1} b_{i-1}}^{\mathrm{MPMAC}}\left(y^{i-1}, x^{i}\right), \\
& =P_{a_{i} b_{i} \mid X^{i} Y^{i-1} a_{i-1} b_{i-1}}^{\mathrm{MPC}}\left(x^{i}, \widehat{y}^{i-1}\right) \sum P_{Y^{i-1} \mid X^{i} a_{i-1} b_{i-1}}^{\mathrm{MPMAC}}\left(y^{i-1}, x^{i}\right), \\
& =P_{a_{i} b_{i} \mid X^{i} Y^{i-1} a_{i-1} b_{i-1}}^{\mathrm{MPMMC}}\left(x^{i}, \widehat{y}^{i-1}\right),
\end{aligned}
$$

where the summations are taken for all $y^{i-1}$ satisfying $b_{i-1}$. From this and Lemma 8, we prove the first claim of Lemma 4. The second follows from the first and the union bound. 